Service social

\title{
La recherche évaluative au profit de la pratique
}

\section{Maryse Rinfret-Raynor, Ginette Larouche et Ann Pâquet-Deehy}

Volume 35, numéro 1-2, 1986

Recherche - Action - Évaluation

URI : https://id.erudit.org/iderudit/706299ar

DOI : https://doi.org/10.7202/706299ar

Aller au sommaire du numéro

Éditeur(s)

École de service social de l'Université Laval

ISSN

1708-1734 (numérique)

Découvrir la revue

Citer cet article

Rinfret-Raynor, M., Larouche, G. \& Pâquet-Deehy, A. (1986). La recherche évaluative au profit de la pratique. Service social, 35(1-2), 141-157.

https://doi.org/10.7202/706299ar d'utilisation que vous pouvez consulter en ligne.

https://apropos.erudit.org/fr/usagers/politique-dutilisation/ 
RINFRET-RAYNOR, Maryse, professeure à l'École de service social de l'Université de Montréal.

LAROUCHE, Ginette, travailleuse sociale au Centre de services sociaux du Montréal Métropolitain.

PÂQUET-DEEHY, Ann, professeure à l'École de service social de l'Université de Montréal.

\section{La recherche évaluative au profit de la pratique *}

\section{Maryse Rinfret-Raynor Ginette Larouche Ann Pâquet-Deehy}

\section{Le concept du praticien-chercheur}

Pratique et recherche, deux concepts perçus par la majorité des praticiens et chercheurs comme peu compatibles : ceux-ci ne font que perpétuer une vision de la pratique et de la recherche qui leur a été transmise durant leur formation en service social. (Hodgins et RinfretRaynor, 1986).

Pour les praticiens, cette incompatibilité se traduit souvent par une faible utilisation dans leur pratique des connaissances rendues disponibles par la recherche (Tripodi et Epstein, 1978; Rosenblatt, 1980). En effet, leur formation les prépare davantage à s'inspirer de leur vécu et à mettre l'accent sur les expériences qu'ils ont connues avec des clients qui présentaient des problèmes similaires. Par ailleurs, Grinnell (1985) suggère qu'il est évident que les travailleurs sociaux accordent davantage d'importance à l'expérience personnelle, la consultation et la supervision qu'aux connaissances qui émergent des recherches. D'autre part, l'expérience pratique s'élabore peu à partir d'un processus systématique de recherche.

L'expérience personnelle suggère, par contre, qu'il y a une certaine appréhension de la part des chercheurs à impliquer des 
praticiens dans une démarche de recherche où ils perçoivent les intervenants comme peu familiers. Les expériences de rechercheaction, quoique représentant un effort pour pallier à cette situation, se butent à des difficultés sérieuses de crédibilité dans le monde universitaire, signifiant ainsi qu'elles sont perçues, non comme une solution acceptable et légitime, mais plutôt comme un palliatif à une situation problématique. Dans le domaine de la recherche empirique, il est intéressant de noter que les relevés sur la littérature concernant les efforts de coopération entre praticiens et chercheurs apportent beaucoup d'informations sur les résistances des premiers à s'impliquer au niveau de la recherche, mais très peu sur celles des seconds à travailler dans une perspective de coopération, avançant ainsi que la responsabilité de la non-coopération appartient davantage aux praticiens qu'aux chercheurs.

En 1973, David Payne compilait une liste des principales raisons qui expliquaient la résistance des gens de la pratique à utiliser ou à supporter les efforts d'évaluation de programmes. Quoique cette liste ait été établie en fonction de la recherche évaluative, les éléments qu'elle comporte peuvent s'appliquer à toute recherche effectuée dans les milieux de services sociaux car ils représentent plusieurs des mythes souvent véhiculés dans ces milieux par les praticiens.

1. Le processus d'évaluation interfère avec celui du développement d'un programme.

2. Il y a conflit d'intérêts entre les membres de l'équipe de recherche et ceux de l'équipe d'intervention.

3. Dans la vision traditionnelle de l'évaluateur, celui-ci est perçu comme devant définir les objectifs du programme.

4. L'évaluation est perçue par plusieurs comme une longue démarche dispendieuse et qui présente peu d'effets positifs à court terme.

5. Par ailleurs, pour certains évaluateurs, l'évaluation n'est acceptable que si elle est composée de corrélations, de tests de signification et d'échantillons effectués au hasard à partir d'une population d'au moins mille personnes.

Dans les milieux de services sociaux, il est possible d'ajouter les éléments suivants :

6. Il y a souvent confusion entre l'évaluation de l'individu et celle d'un programme ou projet dans lequel le praticien est l'acteur principal. La recherche évaluative se préoccupe uniquement des effets du programme. Malheureusement, on a trop souvent confondu les cibles d'évaluation et les praticiens ont été remis en cause par une évaluation montrant de faibles résultats. 
7. Les retombées à court terme sont souvent difficiles à percevoir pour les praticiens car les résultats des évaluations restent la propriété des évaluateurs et des administrateurs. Pour une utilisation maximale des résultats, il est essentiel que les praticiens en soient informés et qu'ils soient, avec les administrateurs, les principaux acteurs dans la prise de décisions qui fait suite à une évaluation.

8. Enfin, plusieurs praticiens sont d'avis que les chercheurs ne connaissent pas assez la pratique pour pouvoir l'évaluer.

Plusieurs auteurs (Scriven, 1967 ; Belasco et Trice, 1969 ; Stufflebeam, 1971, 1985 ; Payne, 1973 ; Grinnell, 1985) ont fait état des avantages de la recherche et de l'évaluation; mentionnons, entre autres, l'aide essentielle que l'évaluation apporte au niveau de la compréhension du processus de traitement, des effets du programme et de la prise de décisions qui émane des résultats de la recherche. Par ailleurs, nous sommes d'avis que l'évaluation est un moyen tangible et exceptionnel de contribuer à l'éthique professionnelle. En effet, comment intervenir auprès du vécu des clients sans s'assurer de la pertinence des interventions par des mesures plus objectives que des perceptions d'analyses basées sur la pratique de l'intervenant?

C'est dans une perspective d'adhésion aux exigences d'éthique professionnelle qu'une praticienne, impliquée dans la recherche d'une intervention efficace auprès des femmes battues, a fait appel à des chercheurs pour poursuivre une démarche déjà entreprise lors d'un processus de formation.

Soucieuse de respecter praticiens et chercheurs dans la démarche de recherche, l'équipe a développé une méthode qui s'apparente au concept du praticien-chercheur tel que développé par Briar (1980) et repris par Grinnell (1985) dans le contexte de la formation en service social.

En effet, dans la perspective de Briar, et suivant les éléments qui définissent le praticien-chercheur, on a formé une équipe préoccupée par le souci d'utiliser et de développer des méthodes de pratique reconnues et démontrées. Celle-ci a constamment été soucieuse d'évaluer sa propre pratique, de participer dans la découverte, la vérification et la publication de méthodes plus efficaces d'aide aux clients. Elle s'est également préoccupée d'utiliser les nouvelles méthodes et approches avec réserve et souci d'évaluation. Désireuse de répondre aux besoins de la pratique, l'équipe a entrepris une démarche ponctuelle de recherche qui devait se poursuivre audelà des objectifs et des délais prévus. 


\section{Interaction entre pratique et recherche}

En complémentarité avec cette recherche, voici le résumé d'une expérience de sept ans sur le développement d'un modèle d'intervention féministe auprès des femmes violentées. Différentes équipes de recherche se sont succédées dans le processus de développement du modèle.

Dans un premier temps, une recherche exploratoire s'élabore afin d'augmenter les connaissances sur la problématique (Hodgins et Larouche, 1980). Cette première démarche permet d'établir l'étiologie du problème et de brosser un tableau des ressources auxquelles font appel les femmes violentées. Les connaissances acquises au cours de la recherche sont par la suite mises à profit dans l'intervention et ont comme conséquence le développement d'une pratique. Un protocole d'intervention témoigne de ce premier acquis dans l'intervention (Larouche, 1981).

Par la suite, une recherche-action vient soutenir l'expérimentation de l'approche de groupe auprès de la même clientèle. Ainsi, un regard se pose sur les notions développées en intervention individuelle et appliquées dans un modèle de groupe (Larouche, Mélançon et Montpetit, 1983). De plus, l'idéologie soutenue dans l'intervention fait l'objet d'une analyse (Lazure, 1983). Suite à cette nouvelle action de la pratique et de la recherche, une ouverture institutionnelle s'établit et permet de poursuivre l'approche de groupe.

Le type d'intervention développé commence à faire l'objet d'échanges dans le réseau des affaires sociales. Des sessions de sensibilisation sont données pour toutes les régions de la province. Une recherche évaluative s'adjoint à l'enseignement afin d'évaluer l'impact de cet enseignement et recueillir de l'information sur l'application du protocole (Pâquet-Deehy, Rinfret-Raynor, Viens et Larouche, 1984). Suite à ces démarches, une nouvelle version du protocole s'avère nécessaire pour développer le cadre conceptuel de l'intervention.

Un guide d'intervention auprès des femmes violentées est alors produit (Larouche, 1985). De plus, la recherche permet de mettre en évidence la pertinence de l'enseignement donné et les intervenantes et les intervenants demandent une formation complète sur le modèle. À cette période-ci de l'évolution de la recherche et de la pratique, une équipe stable praticien-chercheur est établie.

Un programme de formation de dix-huit mois est alors offert dans le cadre d'un cours de perfectionnement de niveau maîtrise à l'Université de Montréal. Une recherche-action s'insère à la formation avec l'objectif d'enrichir le modèle et de le formaliser. L'idéologie féministe fera également l'objet d'une analyse afin d'établir si cette 
dernière a un impact chez les intervenantes suivant la formation. Parallèlement, les effets de cette étape de formation se font sentir dans les milieux de services sociaux. En effet, une certaine formalisation de l'intervention s'opère, et un plus grand nombre d'institutions prennent connaissance de l'intervention prônée par le modèle. Bien que la recherche-action ne soit pas terminée, des pistes se dégagent concernant :

1. le type d'apprentissage ;

2. l'aspect de conscientisation inhérent à la démarche d'intégration des connaissances que suscite le modèle ;

3. les réactions émotives que génère la problématique chez les intervenantes.

Après toutes ces étapes d'évolution, de formalisation et de généralisation du modèle, une recherche évaluative put être entreprise. On a tenté par celle-ci d'établir la pertinence du modèle, de vérifier son efficacité, ses limites et la clientèle cible qu'il rejoignait.

\section{Recherche évaluative}

Rutman (1982) définit la recherche évaluative comme étant "en tout premier lieu, un processus d'application de méthodes scientifiques visant à rassembler des données fiables et valides pour savoir comment et à quel degré des activités particulières produisent des effets ou des résultats particuliers". La définition de Rutman présente un défi intéressant pour le service social, car la recherche du niveau maximal d'exactitude scientifique est soumise aux contraintes d'une pratique sur le terrain. En effet, étant donné que la recherche évaluative constitue un mode de recherche appliquée (Tripodi, 1983), elle est influencée par les mêmes contraintes socio-politiques que la pratique. De plus, étant donné qu'elle porte sur la pratique, elle est dépendante de cette dernière dans son application et non seulement dans sa représentation conceptuelle.

L'évaluation du modèle d'intervention féministe auprès des femmes battues offre l'opportunité d'examiner, dans le cadre de cet article, le défi que présente l'évaluation d'un modèle de pratique.

\section{Préalables à l'évaluation}

La décision d'évaluer un programme présuppose que certains éléments ont été considérés ou sont inhérents au programme 
(Suchman, 1970 ; Rossi, 1972 ; Tripodi, 1983). Par ailleurs, Rutman (1982) affirme que l'évaluation de l'efficacité et de l'efficience d'un programme sous-tend que le programme est articulé, que les objectifs ou résultats sont clairement définis et qu'il y a un lien logique entre le programme, les objectifs et les résultats. La première étape de l'équipe de recherche a donc été l'étude du modèle d'intervention tel que rédigé dans le Guide d'intervention auprès des femmes battues (Larouche, 1985). Cette première étape a favorisé une compréhension relativement cohérente et exacte du modèle. De plus, cette phase préliminaire à l'évaluation a permis de définir, pour les besoins de la recherche, le concept d'«idéologie féministe" et le modèle qui s'en inspire.

Le modèle d'intervention rejoint, de fait, les préoccupations de l'idéologie féministe. Ce cadre d'analyse permet de situer la violence dans son aspect socio-politique (Ball et Wyman, 1977-78). Le processus de conscientisation fait ainsi partie de la démarche d'intervention et amène les femmes à prendre conscience que leurs difficultés sont engendrées par leur condition de femme (Kravetz, 1976). De plus, le regroupement des femmes sert de moyen pour que celles-ci reprennent du pouvoir et se solidarisent (Carden, 1974). Ce processus socio-politique de l'intervention évolue dans un cadre institutionnel. Cette perspective d'action rejoint donc l'idéologie des féministes réformistes (Corbeil et al., 1983).

Le modèle proposé suggère différentes techniques d'intervention provenant de plusieurs écoles de pratique. Toutefois, un grand nombre de ces techniques appartiennent à l'école behavioriste (entraînant à l'affirmation de soi) (Jakubowski, 1973, 1977). Les séquences d'intervention définies à partir de l'évolution de la situation de violence nécessitent une action prônée par un modèle spécifique à la crise. Ainsi, diverses techniques relèvent de l'approche planifiée à court terme (CareyBélanger, 1974).

Des notions de l'analyse transactionnelle (Harris, 1973) sont également intégrées. Il en est de même pour l'approche corporelle où certaines techniques de bioénergie sont employées (Lowen, 1970). L'intervention de groupe fait partie intégrante du modèle comme stratégie d'intervention. Les groupes de conscientisation (Kravetz, 1976) et d'entraînement à l'affirmation de soi servent de référence (Jansen et Meyers-Abell, 1981).

Un aspect important demeure le suivi distinct de la victime et de l'agresseur. Le travail s'effectue par contrats de douze rencontres renégociables. L'utilisation de tâches spécifiques permet de responsabiliser la clientèle et le travail s'effectue avec un réseau de collaborateurs afin d'assurer l'aide concrète (Larouche, 1985). 
Ayant spécifié le modèle d'intervention et son idéologie, une deuxième étape a été d'étudier ses objectifs d'intervention auprès des femmes battues. Cette étude a permis, d'une part, de constater que les objectifs étaient suffisamment clairs et articulés pour pouvoir procéder à l'évaluation et, d'autre part, d'identifier les objectifs spécifiques: l'élimination de la violence conjugale, l'amélioration du mode de résolution des conflits de couple, l'augmentation du contrôle personnel, la diminution du sentiment de culpabilité, l'augmentation du bien-être personnel, l'augmentation de l'autonomie, l'amélioration de l'ajustement conjugal, l'amélioration de l'ajustement psychosocial, l'augmentation de l'estime de soi et, enfin, l'amélioration de l'assertion dans le comportement général et face au conjoint. Cette étape a été une phase très importante dans l'évaluation effectuée avant d'entreprendre la recherche évaluative car elle constituait la base à partir de laquelle toutes les variables indépendantes allaient être spécifiées. Rutman (1982) ajoute que le processus de particularisation des objectifs garantit que les variables-clés ont été identifiées et qu'elles seront incluses dans l'évaluation. Sans des objectifs clairs et opérationnalisés, il est impossible de réaliser une recherche évaluative.

Une troisième étape consiste à vérifier le lien logique entre les activités du traitement et les objectifs. Une revue de l'expérience des praticiennes qui travaillent avec le modèle d'intervention féministe auprès des femmes battues a permis à l'équipe de recherche de présumer qu'un lien logique existe entre le traitement et les objectifs.

Deux autres facteurs ont été considérés avant d'entreprendre la recherche évaluative. Un premier élément a été celui des ressources financières. À cet égard, un projet de recherche a été soumis aux Subventions nationales de bien-être, à Ottawa, et une importante subvention a été obtenue.

L'usage et l'utilisation des résultats de la recherche ont été un deuxième et dernier élément qui a été considéré avant d'entreprendre l'évaluation du modèle d'intervention féministe auprès des femmes battues. Cette question a été traitée avec beaucoup d'attention car les résultats de la recherche avaient essentiellement pour but d'apporter de l'information afin d'enrichir le modèle d'intervention et de le rendre le plus conforme possible aux besoins des femmes battues. Dans cette perspective, il fut décidé que les résultats devraient être accessibles, non seulement aux chercheurs et organismes subventionnaires, mais également à l'ensemble des intervenants et intervenantes travaillant auprès des femmes violentées. 


\section{Objectifs du projet de recherche}

Le projet de recherche a comme objectif principal d'évaluer l'efficacité du modèle d'intervention féministe auprès des femmes battues tel qu'il est décrit dans le Guide d'intervention auprès des femmes violentées (Larouche, 1985). Cet objectif s'inscrit dans l'optique d'une évaluation de programme telle que définie par Rutman (1982) et ayant comme objet d'établir les conséquences des interventions d'un programme. Par ailleurs, l'efficacité de cette intervention sera principalement définie ici par l'arrêt de la violence conjugale ou par la renégociation des rôles conjugaux. Les autres variables dépendantes correspondront aux objectifs de l'intervention féministe auprès de femmes battues tels qu'ils ont été présentés précédemment.

Le projet de recherche dont il est fait mention ici vise également deux objectifs secondaires reliés à la réalisation d'une étude prédictive. Grinnell (1985) définit une étude prédictive comme servant à identifier une variété de facteurs pouvant prédire un phénomène. Dans ce contexte, un deuxième objectif de la recherche consiste à analyser certains facteurs susceptibles d'être associés à la poursuite ou à l'abandon de la thérapie. Enfin, un troisième objectif consistera à explorer comment des facteurs, autres que le traitement, contribuent à l'élimination de la violence. L'étude prédictive sera de nature exploratoire étant donné le peu de connaissances existantes sur les traitements spécifiques auprès des femmes victimes de violence. Les variables reliées à l'étude prédictive sont déterminées à partir de la documentation clinique et expérimentale. Comme le soulignent Bergin et Lambert (1978), il convient de répondre à la question suivante : quelle thérapie est efficace, auprès de quelle clientèle, et quel type de thérapeute est le plus en mesure de la dispenser?

\section{Méthodologie de la recherche}

\section{Sujets}

Les sujets rejoints sont des femmes ayant subi de la violence physique au cours des trois mois précédant une demande d'aide aux différents C.S.S. et C.L.S.C. de l'ensemble du Québec. Pour les fins de la recherche, une femme battue sera définie comme une femme dont le partenaire de sexe opposé a utilisé la force physique envers elle. Nous étudions présentement quelles seraient les conséquences méthodologiques d'inclure également dans la recherche les femmes qui ont été menacées de violence physique durant cette période. 
Les femmes sont d'abord sélectionnées en fonction de leur demande d'aide. Ainsi, toutes celles qui demandent de l'aide suite à un vécu de violence conjugale peuvent participer à la recherche. De plus, étant donné que peu consultent pour des situations de violence, l'équipe de recherche souhaite que, suite à des consultations auprès des établissements concernés, un instrument de dépistage soit utilisé à l'accueil auprès de toutes les femmes vivant avec un conjoint et qui font une demande d'aide.

Suite à l'entrevue d'accueil, toutes les femmes ayant expérimenté un vécu de violence et qui ne démontrent pas de désordre schizophrénique (Rounsaville, Lipton et Bieber, 1978; Goodstein et Page, 1981) sont orientées de façon aléatoire vers une praticienne du groupe expérimental ou du groupe contrôle. Il est entendu que, si la femme arrive en état de crise, elle reçoit d'abord les services d'urgence nécessaires.

À la première entrevue avec l'intervenante, la cliente complète une fiche signalétique sur les caractéristiques socio-démographiques et cliniques, et elle est informée qu'une recherche concernant sa situation est présentement en cours. Son aide est sollicitée et les détails concernant sa participation à ce projet lui sont communiqués. Après cette première entrevue, l'intervenante transmet au directeur de la recherche le nom des clientes qui acceptent de participer au projet. Celles qui refusent de participer à la recherche continuent d'être suivies, indépendamment de la recherche. Par ailleurs, une assistante de recherche, formée en intervention psychosociale, visite les femmes ayant accepté de participer et les aide à remplir les divers instruments de mesure utilisés. Les rencontres consistent en une entrevue semistructurée. Ce choix a été fait afin de tenir compte du temps nécessaire pour remplir les questionnaires, et aussi parce que la plupart des femmes qui cherchent de l'aide dans le réseau institutionnel sont généralement peu scolarisées (Hodgins et Larouche, 1981).

Le processus de sélection se déroulera sur une période de six mois afin d'obtenir le nombre total de deux cent sujets. Tous les efforts sont faits afin de rencontrer les femmes qui participent à la recherche à l'intérieur des sept jours qui suivent.

\section{Devis expérimental}

Afin de mesurer l'impact de cette intervention féministe et spécifique auprès des femmes battues, un schème expérimental avec groupe contrôle est utilisé. Plus spécifiquement, il s'agit d'une étude comparative des effets du traitement psychosocial reçu par trois 
groupes de femmes victimes de violence conjugale. Ces trois groupes sont constitués de la façon suivante: un premier groupe de sujets bénéficiant d'une thérapie de groupe selon le modèle d'intervention féministe ; un deuxième bénéficiant d'une thérapie individuelle selon le même modèle; et enfin un troisième bénéficiant d'une thérapie selon l'approche habituelle. Les sujets du premier groupe sont sélectionnés suite à une période de recrutement offrant spécifiquement une intervention féministe de groupe à des femmes victimes de violence de la part de leur partenaire. Les sujets des groupes deux et trois sont attribués au hasard à un des deux types de traitement.

Les intervenantes des groupes un et deux ont été formées à l'intervention féministe auprès des femmes battues. Celles du groupe trois furent sélectionnés dans chaque milieu où intervenaient les participantes de la formation. De plus, les intervenantes du groupe contrôle furent choisies en fonction de leur formation, des caractéristiques de leur expérience et de leur formation professionnelle comparables à celles des intervenantes du groupe expérimental.

Des mesures furent prises avant et après le traitement ainsi qu'à trois reprises par la suite. L'intervalle entre chaque mesure fut de six mois. Selon Campbell et Stanley (1966) ce schème est bien contrôlé. En effet, il permet de déterminer les effets globaux du traitement en contrôlant tout changement qui pourrait survenir chez le groupe expérimental autre que celui produit par l'intervention. Pour des raisons d'éthique professionnelle, le groupe contrôle avec traitement habituel a été choisi plutôt que le groupe témoin, le groupe contrôle permettant de fournir un traitement à tous les sujets participant à la recherche. Cette décision respecte l'acte professionnel qui oblige de fournir un traitement à toute personne qui en fait la demande. Ceci exclut automatiquement l'utilisation d'un groupe témoin traditionnel. Par ailleurs, le choix de ce groupe contrôle permet d'étudier les effets différentiels de l'intervention proposée en comparaison à un traitement régulièrement dispensé dans les établissements de services sociaux.

En accord avec les objectifs de la recherche présentés précédemment, outre la variable indépendante, c'est-à-dire le traitement reçu, trois grandes catégories de variables seront étudiées: dépendantes, concomitantes ou de contrôle et prédictives.

Les variables dépendantes ont été choisies en fonction des trois niveaux concernés par les résultats thérapeutiques, selon le modèle tripartite de Hadley et Strupp (1977) : l'organisme ou la société, les clientes et l'intervention. Ainsi, les effets du traitement auprès des femmes battues sont mesurés, d'une part, par l'élimination de la 
violence conjugale manifestée par l'amélioration de l'ajustement conjugal ou la séparation des conjoints. Par ailleurs, l'intervention féministe possède certains objectifs supplémentaires qui serviront également d'indicateurs des effets du traitement: l'amélioration du contrôle personnel des clientes, la diminution de leur sentiment de culpabilité, l'augmentation de leur bien-être personnel, de leur autonomie, de leur ajustement psychosocial, de leur estime de soi, de leur assertion générale et face à leur conjoint, de leur ajustement conjugal, et des modes de résolution des conflits de couple.

Les variables contrôles sont, par ailleurs, étudiées en fonction de la relation cliente-thérapeute. Pour les clientes elles-mêmes, l'étude tient compte du diagnostic clinique, des événements de vie concomitants au traitement, du support social reçu avant et pendant le traitement, de la nature et de la motivation de la demande d'aide, de leurs attitudes envers les rôles féminins et de leur perception de la qualité de la relation thérapeutique. Quant aux thérapeutes, l'analyse portera sur leurs attentes quant au traitement, leurs valeurs et attitudes, leur expérience de pratique et leur formation spécifique.

Les variables prédictives ont été sélectionnées à partir d'études antérieures ayant déjà permis d'en identifier quelques-unes ou d'émettre des hypothèses à partir d'entrevues cliniques. L'étude prédictive portera sur deux catégories de facteurs; l'une associée à la poursuite de la thérapie et l'autre à l'élimination de la violence conjugale, indépendamment du traitement. Afin de réaliser l'étude prédictive de la poursuite ou de l'abandon de la thérapie, les mesures s'effectueront en fonction, d'abord, de l'influence des variables démographiques, sociales et économiques. De plus, les caractéristiques concernant la demande, la violence subie, les facteurs psychologiques (l'espoir de changer sa situation, le degré de peur de la violence du conjoint, le niveau d'anxiété) et l'attitude envers les rôles féminins seront analysés. L'étude prédictive de l'élimination de la violence comportera d'abord l'analyse des caractéristiques démographiques, sociales et économiques, puis de celles reliées à la violence subie, à la nature de l'aide reçue à l'extérieur du réseau, et enfin des caractéristiques psychologiques comprises dans la volonté de la cliente de quitter son conjoint. Finalement, l'espoir de pouvoir changer sa situation, les attitudes des clientes envers les rôles féminins et la peur qu'elles éprouvent d'être tuées seront intégrés à l'étude. 


\section{Phases critiques pour la réalisation de la recherche}

La réalisation de la recherche évaluative telle que présentée dans cet article représente un défi pour les chercheurs et les praticiens. Dans cette perspective, certains facteurs apparaissent comme particulièrement critiques par rapport à la réalisation du projet. Un premier élément de préoccupation concerne le choix des instruments de mesure. Le repérage effectué à date nous permet de constater les phénomènes suivants: il existe peu d'instruments de langue française reliés au domaine de recherche sur les femmes violentées; ceux de langue anglaise ont souvent été validés avec des populations de niveau socioéconomique moyen, alors que les sujets de la présente étude proviennent principalement d'un milieu socio-économiquement faible; et enfin les instruments ne sont pas adaptés à des populations féminines.

Un deuxième élément crucial est celui de la négociation sur le terrain, qui a pour but d'établir un protocole d'entente concernant l'implication des intervenantes, la sélection et le recrutement de sujets. Cette phase est particulièrement importante puisqu'elle influence directement le devis de recherche, amène le chercheur à adapter le modèle "idéal" de recherche aux contraintes de la pratique. Dans l'étude du modèle d'intervention auprès des femmes battues, les différences régionales présentent des éléments dont il faut absolument tenir compte. Rutman (1982) rappelle que c'est dans le cadre de telles contraintes que chercheurs et praticiens s'appliquent à obtenir le maximum de rigueur pour résoudre des problèmes méthodologiques de base tels que la fiabilité, la validité, l'échantillonnage, la répartition au hasard, le plan de recherche et l'analyse des données.

Un troisième élément de préoccupation est relié à l'identification des femmes battues comme sujets d'étude. En effet, même si la société est de plus en plus consciente de la violence faite aux femmes, des difficultés demeurent dans le réseau institutionnel pour dépister et identifier cette clientèle (Larouche et Hodgins, 1981). De plus, il existe peu de connaissances sur le nombre de femmes qui consultent les services sociaux spécifiquement pour obtenir de l'aide pour une situation de violence conjugale. En effet, souvent le problème de la violence est identifié par le biais d'une autre problématique telle les cas de protection de la jeunesse. Dans cette perspective, certains problèmes concernant le recrutement des sujets dans la période prévue de six mois peuvent représenter une difficulté. En vue de pallier partiellement à cette situation, il est prévu de prolonger la période de 
six mois à neuf mois afin d'augmenter les probabilités de recruter les deux cents sujets souhaités pour l'étude.

Enfin, une quatrième préoccupation est la " mortalité expérimentale " ou la perte de sujets durant la recherche. L'étude prévoit suivre les sujets sur une période de deux ans, ce qui implique de pouvoir les retracer, non seulement au post-test, qui aura lieu immédiatement à la fin du traitement, mais également aux périodes de relance prévues par le projet. Par ailleurs, l'intervention auprès des femmes battues aide ces dernières à développer des mécanismes de protection qui impliquent souvent le déménagement et la relocalisation de la cliente dans un nouveau milieu. Cette situation a amené l'équipe de recherche à se pencher sur cette question afin d'assurer qu'un nombre minimal de sujets participe à la troisième relance afin de procéder à des analyses statistiques pertinentes.

Il est évident que, malgré les difficultés anticipées présentement, d'autres viendront s'ajouter à la liste de celles que l'équipe a déjà identifiées. Par ailleurs, n'est-ce pas un défi pour la recherche de pouvoir transiger avec les réalités de la pratique tout en maintenant un minimum de rigueur scientifique ? Cette fine ligne d'équilibre entre les exigences de la pratique et celles de la recherche ne peut pas être maintenue sans la coopération et le respect mutuel des chercheurs et des praticiens.

\section{Conclusion}

Au terme de cette réflexion, nous pouvons constater que la recherche joue un rôle important dans le développement d'un modèle d'intervention. Plusieurs formes de recherches peuvent, dans ce sens, contribuer au développement du modèle et chacune d'elle agit comme un élément de support en fournissant des données qui sous-tendent le modèle et permettent de le faire avancer. La recherche évaluative se situe comme dernière étape dans le processus de développement du modèle. Elle vient en fixer les limites et les capacités. Dans cette perspective, elle prend son sens en autant que le modèle est stable, généralisé, suffisamment articulé et opérationnalisé pour en permettre l'enseignement.

Par ailleurs, l'implication de praticiens et de chercheurs dans une équipe de recherche évaluative, dont le but principal est de supporter le développement d'un modèle d'intervention, présente des avantages et des désavantages pour chacun des acteurs. 
En ce qui concerne les désavantages, un certain nombre d'éléments peuvent être identifiés. Un premier domaine de difficultés est relié à la compétition qui risque de s'établir entre les membres de l'équipe. Celle-ci se manifeste non seulement entre les praticiens et les chercheurs mais également, et peut-être davantage, entre les membres représentant le même regroupement, c'est-à-dire praticiens ou chercheurs. En effet, chacun apporte à l'équipe ses attentes, ses aspirations et son idéologie, qui lui servent de cadre d'analyse chaque fois qu'une situation se présente. Dans ce contexte, les zones de conflit demeurent minimalement constantes et latentes, indépendamment des intérêts et de la rentabilité que la démarche représente pour chacun.

La négociation des exigences de la pratique et de la recherche constitue un deuxième secteur de difficulté. En effet, bien que cette étape constitue une des richesses d'allier pratique et recherche, elle représente également une pierre d'achoppement pour les membres de l'équipe de recherche, chacun pouvant demeurer limité par sa sphère d'analyse.

Si ce mode de fonctionnement comporte plusieurs désavantages, il représente également plusieurs avantages qui rendent l'expérience valable et pertinente. Parmi ceux-ci, mentionnons tout d'abord la stimulation intellectuelle apportée par ce mode de fonctionnement. En effet, les connaissances diverses de chaque membre de l'équipe favorisent l'expansion du schème de référence de chacun. De plus, l'analyse différente faite par chacun stimule la confrontation intellectuelle et permet aux individus de vérifier leurs limites, leurs connaissances et leurs zones de compétence.

De plus, le fonctionnement en équipe avec des personnes représentant des orientations différentes motive chacun à nommer et à reconnaître son "non-dit", c'est-à-dire le contenu, schème de référence ou valeur que chacun considère acquis mais que les autres ne connaissent pas. Un troisième avantage est relié aux bénéfices que ce processus apporte aux chercheurs. En effet, il leur permet d'entrer en contact avec la réalité de la pratique et de mettre à l'épreuve sur le terrain les modèles théoriques qu'il défend. De plus, cette expérience fournit aux chercheurs l'opportunité de développer une connaissance de la pratique qui peut être utilisée pour enrichir son enseignement.

Par ailleurs, la participation à une équipe de recherche centrée sur l'enrichissement d'un modèle d'intervention offre la possibilité aux praticiens de systématiser leur pratique et de la rendre crédible dans les milieux d'intervention. En effet, le processus de recherche leur apporte des données objectives dont ils peuvent s'inspirer pour développer et 
enrichir des modes d'interventions auprès d'une population donnée et ainsi, suivant le modèle du praticien-chercheur (Briar, 1981), participer à la découverte, la vérification et la diffusion d'un modèle plus efficace pour aider les clients.

Disons, enfin, que la mise en commun des connaissances de la pratique et de la recherche fournit une occasion de faire avancer la profession et de développer des connaissances systématiques pour l'intervention.

\section{Note}

* Les projets de recherche évaluative et celui de recherche-action dont il est fait mention dans ce texte ont fait l'objet de subventions de la part de Subventions nationales de bien-être (Ottawa).

\section{Références bibliographiques}

BALL, P.G. et E. WYMAN, " Battered wives and powerlesness : what councelors do" ", Victimology: An International Journal, vol. 2, no 3-4, 1977-78: 545-552.

Belasco, J.A. et H.M. Trice, The Assessment of Change in Training and Therapy, New York, McGraw-Hill, 1969.

BERGIN, A.E. et M.J. LAMBERT, "The evaluation of therapeutic outcomes", dans: S.L. Garfield et A.E. Bergin (éds), Handbook of Psychotherapy and Behavior Change, New York, Wiley, 1978: 139-190.

BRIAR, S., "Toward integration at practice and research", dans: D. FANSHEL (éd.), Future of Social Work Research, Washington (D.C.), National Association of Social Workers, 1980 : 31-37.

BRIAR, S., "The project on research utilization in social work education", dans : S. Briar, H. Weissman et A. Rubin (éds), Research Utilization in Social Work Education, New York, Council on Social Work Education, 1981: 1-5.

CAMPBell, D.T. et J.C. Stanley, Experimental and Quasi Experimental Designs for Research, Chicago, Rand McNally, 1966.

Carden, N.L., The New Feminist Movement, New York, Russel Sage Foundation, 1974.

CAREY-BÉlAnger, E. "Le traitement à court terme: ses techniques et ses stratégies ", Service social, vol. 23, no 2 et 3, 1974: 57-68.

Corberl, C. et al., L'intervention féministe: l'alternative des femmes au sexisme en thérapie, Montréal, Les Éditions coopératives Albert St-Martin, 1983.

Goodstein, R.K. et A.W. PAGE, "Battered wife syndrome: Overview of dynamics and treatment", American Journal of Psychiatry, vol. 138, no 8, 1981 : 1036-1044. 
GrinnelL, R.M., Social Work Research and Evaluation, Itasca (III.), Peacock, 1985.

Hadley, S. et H.H. Strupp, "A tripartite model of mental and therapeutic outcomes ", American Psychologist, vol. 32, no 3, 1977 : 187-196.

Harris, T.A., I'm Ok - You're Ok. New York, Avon, 1973.

Hodgins, S. et G. LAROUCHE, Violence conjugale : antécédents et conséquences, Ottawa, Bureau du Solliciteur général du Canada, 1981.

HODGINS, S. et M. RINFRET-RAYNOR, "Social work practice and research : are they incompatible", Revue canadienne de service social, (à paraître, 1986).

JAKUBOWSKI, S.P., "Facilitating the growth of women through assertive training ", Counseling Psychology, vol. 4, no 1, 1973: 75-86.

JAKUBOWSKI, S.P., "Self-assertion training procedures for women", dans: E. Rawlings et D. CARTer (éds), Psychotherapy for Women: Treatment Toward Equality. Springfield, III., Charles C. Thomas, 1977 : 168-192.

JANSEN, M.A. et J.N. MeYers-ABeLL, "Assertive training for battered women and a pilot program ", Social Work, vol. 26, no 2, 1981: 164-165.

KRAVETZ, D.F., "Consciousness raising groups and group psychotherapy: alternative mental health resources for women ", Psychotherapy, Theory, Research and Practice, vol. 13, no 1, 1976: 66-71.

LAROUCHE, G., Guide d'intervention auprès des femmes violentées, Montréal, Corporation professionnelle des travailleurs sociaux du Québec, 1985.

Larouche, G., B. Mélançon et L. MontPetit, Projet de groupe auprès des femmes violentées, Montréal, document déposé au C.S.S.M.M., 1982.

Lazure, C., Étude thématique, Université de Montréal, École de service social, 1983.

LOWEN, A., La bioénergie, Montréal, Jour/Tchou, 1970.

Pâquet-Deehy, A., M. Rinfret-Raynor, L. Viens et G. Larouche, Évaluation des connaissances acquises par les intervenants sociaux suite à une session de formation sur la problématique des femmes violentées. Rapport de recherche, École de service social, Université de Montréal, 1984.

PAYNE, D.A., "Some old and new wives tales concerning curriculum evaluation", Educational Leadership, vol. 30, no 4, 1973: 343-347.

ROSENBLATT, A., "The practitioner's use and evaluation of research ", Social Work, vol. 13, no 1, $1968: 53-59$.

Rossı, P.H., "Booby traps and pitfalls in evaluation of social action programs", dans: C.H. Welss (éd.), Evaluating Action Programs: Readings in Social Action and Education, Boston, Allyn and Bacon, 1972: 224-236.

ROUNSAVILLE, B., N. LIPTON et M. BIEBER, "The natural history of a psychotherapy group for battered women ", Psychiatry, vol. 42, n० 1, $1978: 63-78$.

RUtMAN, L., "Planification d'une étude évaluative", dans: R. LeCOMTE et L. RUTMAN (éds), Introduction aux méthodes de recherche évaluative, Québec, Les Presses de l'Université Laval, 1982: 23-46.

SCRIVEN, M., (1967). "The methodology of evaluation", dans : R.W. TYLER, R.W. GAGNé et M. SCRIven (éds), Perspectives of Curriculum Evaluation, A.E.R.A. Monograph Series on Curriculum Evaluation, n 1, pp. 39-83. 
StUfFLEBEAM, D.L., "The relevance of the CIPP evaluation model for educational accountability", Journal of Research and Development in Education, 1, 1971 : 19-25.

StufflebeAM, D.L., Conférence prononcée dans le cadre du congrès de la société canadienne en évaluation, Calgary, Canada, 1985.

SUCHMAN, E.A., "Action for what ? A critique of evaluation research", dans : C.H. WeIsS (éd.), Evaluating Action Programs: Readings in Social Action and Education, Boston, Allyn and Bacon, 1972: 52-85.

TRIPODI, T., Evaluative Research For Social Workers, Englewood Cliffs (N.J.), Prentice-Hall, 1983.

TRIPODI, T. et I. EPSTEIN, «Incorporating knowledge of research methodology into social work practice ", Journal of Social Service Research, 2, 1978 : 11-23. 